\title{
Health Monitoring and Management Using Internet-of-Things (IoT) Sensing with Cloud-based Processing: Opportunities and Challenges
}

\author{
Moeen Hassanalieragh*, Alex Page*, Tolga Soyata*, Gaurav Sharma*, Mehmet Aktas ${ }^{\dagger}$, Gonzalo Mateos* \\ Burak Kantarci $i^{\ddagger}$, Silvana Andreescu ${ }^{\S}$ \\ ${ }^{*}$ Dept. of ECE, ${ }^{\dagger}$ URMC \\ University of Rochester \\ Rochester, NY 14627 \\ \{mhassan3,apage4,soyata,sharma,gmateosb\}@ece.rochester.edu \\ Mehmet_Aktas@urmc.rochester.edu \\ ${ }^{\ddagger}$ Dept. of ECE, ${ }^{\S}$ Chem \& BioM Sci \\ Clarkson University \\ Postdam, NY 13699 \\ \{bkantarc,eandrees\}@ clarkson.edu
}

\begin{abstract}
Among the panoply of applications enabled by the Internet of Things (IoT), smart and connected health care is a particularly important one. Networked sensors, either worn on the body or embedded in our living environments, make possible the gathering of rich information indicative of our physical and mental health. Captured on a continual basis, aggregated, and effectively mined, such information can bring about a positive transformative change in the health care landscape. In particular, the availability of data at hitherto unimagined scales and temporal longitudes coupled with a new generation of intelligent processing algorithms can: (a) facilitate an evolution in the practice of medicine, from the current post facto diagnose-andtreat reactive paradigm, to a proactive framework for prognosis of diseases at an incipient stage, coupled with prevention, cure, and overall management of health instead of disease, (b) enable personalization of treatment and management options targeted particularly to the specific circumstances and needs of the individual, and (c) help reduce the cost of health care while simultaneously improving outcomes. In this paper, we highlight the opportunities and challenges for IoT in realizing this vision of the future of health care.
\end{abstract}

Keywords-remote health monitoring; IoT; visualization; analytics;

\section{INTRODUCTION}

Recent years have seen a rising interest in wearable sensors and today several devices are commercially available [1]-[3] for personal health care, fitness, and activity awareness. In addition to the niche recreational fitness arena catered to by current devices, researchers have also considered applications of such technologies in clinical applications in remote health monitoring systems for long term recording, management and clinical access to patient's physiological information [4]-[8]. Based on current technological trends, one can readily imagine a time in the near future when your routine physical examination is preceded by a two-three day period of continuous physiological monitoring using inexpensive wearable sensors. Over this interval, the sensors would continuously record signals correlated with your key physiological parameters and relay the resulting data to a database linked with your health records. When you show up for your physical examination, the doctor has available not only conventional clinic/lab-test based static measurements of your physiological and metabolic state, but also the much richer longitudinal record provided by the sensors. Using the available data, and aided by decisionsupport systems that also have access to a large corpus of observation data for other individuals, the doctor can make a much better prognosis for your health and recommend treatment, early intervention, and life-style choices that are particularly effective in improving the quality of your health. Such a disruptive technology could have a transformative impact on global healthcare systems and drastically reduce healthcare costs and improve speed and accuracy for diagnoses.

Technologically, the vision presented in the preceding paragraph has been feasible for a few years now. Yet, wearable sensors have, thus far, had little influence on the current clinical practice of medicine. In this paper, we focus particularly on the clinical arena and examine the opportunities afforded by available and upcoming technologies and the challenges that must be addressed in order to allow integration of these into the practice of medicine. The paper is organized as follows: Section II highlight some of the key related work in this area. In Section III, we outline the architecture for remote health monitoring systems based on wearable sensors, partitioning the system into for main components acquisition, analytics, and visualization. In Sections IV- VII we highlight the opportunities and challenges related to each of these components. We conclude the paper in Section VIII with a summary and discussion.

\section{BACKGROUND}

Most proposed frameworks for remote health monitoring leverage a three tier architecture: a Wireless Body Area Network (WBAN) consisting of wearable sensors as the data acquisition unit, communication and networking and the service layer [4], [7]-[10]. For instance [11] proposes a system that recruits wearable sensors to measure various physiological parameters such as blood pressure and body temperature. 
Sensors transmit the gathered information to a gateway server through a Bluetooth connection. The gateway server turns the data into an Observation and Measurement file and stores it on a remote server for later retrieval by clinicians through the Internet. Utilizing a similar cloud based medical data storage, a health monitoring system is presented in [12] in which medical staff can access the stored data online through content service application. Targeting a specific medical application, WANDA [13] an end to end remote health monitoring and analytics system is presented for supervision of patients with high risk of heart failure.

In addition to the technology for data gathering, storage and access, medical data analysis and visualization are critical components of remote health monitoring systems. Accurate diagnoses and monitoring of patient's medical condition relies on analysis of medical records containing various physiological characteristics over a long period of time. Dealing with data of high dimensionality in both time and quantity makes data analysis task quite frustrating and error prone for clinicians. Although the use of data mining and visualization techniques had previously been addressed as a solution to the aforementioned challenge [14], [15], these methods have only recently gained attention in remote health monitoring systems [16], [17].

While the advent of electronic remote health monitoring systems has promised to revolutionize the conventional health care methods, integrating the IoT paradigm into these systems can further increase intelligence, flexibility and interoperability [9], [18]. A device utilizing the IoT scheme is uniquely addressed and identifiable at any time and anywhere through the Internet. IoT based devices in remote health monitoring systems are not only capable of the conventional sensing tasks but can also exchange information with each other, automatically connect to and exchange information with health institutes through the Internet, significantly simplifying set up and administration tasks. As exemplified in [19], such systems are able to provide services such as automatic alarm to the nearest healthcare institute in the event of a critical accident for a supervised patient.

\section{SySTEM ARCHITECTURE}

Figure 1 illustrates the system architecture for a remote health monitoring system, whose major components we describe next:

Data Acquisition is performed by multiple wearable sensors that measure physiological biomarkers, such as ECG, skin temperature, respiratory rate, EMG muscle activity, and gait (posture). The sensors connect to the network though an intermediate data aggregator or concentrator, which is typically a smart phone located in the vicinity of the patient.

The Data Transmission components of the system are responsible for conveying recordings of the patient from the patient's house (or any remote location) to the data center of the Healthcare Organization (HCO) with assured security and privacy, ideally in near real-time. Typically, the sensory acquisition platform is equipped with a short range radio such

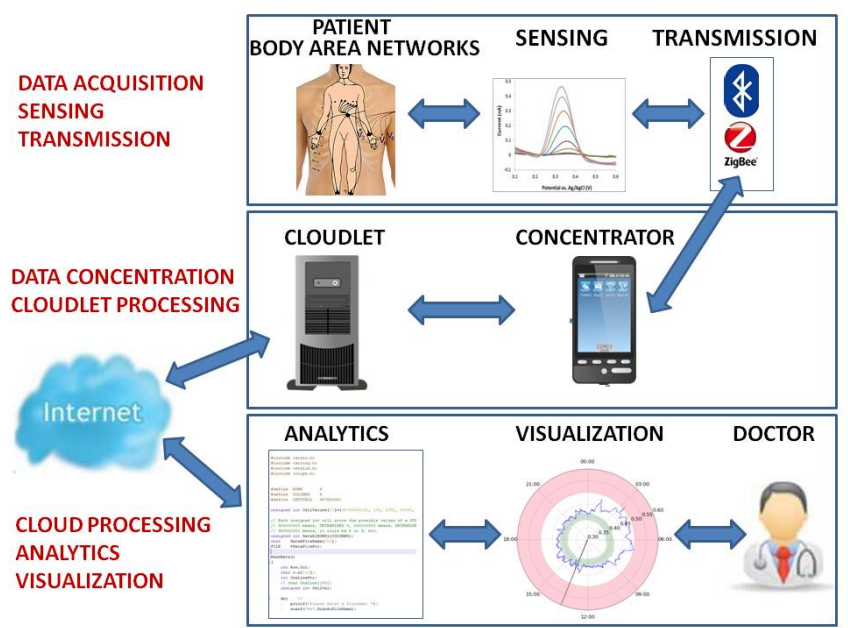

Fig. 1. Components of a remote patient monitoring system that is based on an IoT-Cloud architecture.

as Zigbee or low-power Bluetooth, which it uses to transfer sensor data to the concentrator. Aggregated data is further relayed to a $\mathrm{HCO}$ for long term storage using Internet connectivity on the concentrator, typically via a smartphone's WiFi or cellular data connection. Sensors in the data acquisition part form an Internet of Things (IoT)-based architecture as each individual sensor's data can be accessed through the Internet via the concentrator [20], [21].

Often a storage/processing device in vicinity of a mobile client, sometimes referred to as a cloudlet, is used to augment its storage/processing capability whenever the local mobile resources do not fulfill the application's requirements [22]. The cloudlet can be a local processing unit (such as a desktop computer) which is directly accessible by the concentrator through WiFi network. In addition to providing temporary storage prior to communication of data to the cloud, the cloudlet can also be used for running time critical tasks on the patient's aggregated data. Moreover, the cloudlet can be used to transmit the aggregated data to the cloud in case of limitations on the mobile device such as temporary lack of connectivity or energy.

Cloud Processing has three distinct components: storage, analytics, and visualization. The system is designed for long term storage of patient's biomedical information as well assisting health professionals with diagnostic information. Cloud based medical data storage and the upfront challenges have been extensively addressed in the literature [23], [24]. Analytics that use the sensor data along with e-Health records that are becoming prevalent can help with diagnoses and prognoses for a number of health conditions and diseases. Additionally, Visualization is a key requirement for any such system because it is impractical to ask physicians to pore over the voluminous data or analyses from wearable sensors. Visualization methods that make the data and analyses accessible to them in a readily digestible format are essential if the wearable sensors are to impact clinical practice.

In the following sections, we consider the key elements 


\begin{tabular}{|c|c|c|c|c|}
\hline Bio-marker & CVD & COPD & PD/HD & Diabetes \\
\hline \hline Gait (posture) & $\checkmark \checkmark$ & $\checkmark \checkmark$ & $\checkmark \checkmark$ & $?$ \\
ECG & $\checkmark \checkmark$ & $\checkmark \checkmark$ & $\checkmark$ & $?$ \\
Respiratory rate & $\checkmark \checkmark$ & $\checkmark \checkmark$ & $\checkmark$ & $?$ \\
Skin temperature & $\checkmark$ & $\checkmark$ & $\checkmark$ & $\checkmark$ \\
Surface EMG & $\checkmark$ & $\checkmark$ & $\checkmark$ & $?$ \\
Sweating & $?$ & $?$ & $\checkmark$ & $?$ \\
Blood pressure & $\checkmark \checkmark$ & $\checkmark$ & $\checkmark$ & $\checkmark$ \\
Body movements & $\checkmark$ & $?$ & $\checkmark \checkmark$ & $?$ \\
Blood Glucose & $?$ & $?$ & $?$ & $\checkmark \checkmark$ \\
\hline Heart Sound & $\checkmark$ & $\checkmark$ & $?$ & $?$ \\
Oxygenation & $\checkmark \checkmark$ & $\checkmark \checkmark$ & $?$ & $?$ \\
Title volume & $\checkmark \checkmark$ & $\checkmark \checkmark$ & $\checkmark$ & $?$ \\
\hline
\end{tabular}

TABLE I

LIST OF AVAILABLE (TOP) AND FUTURE (BOTTOM) SENSORS AND THEIR APPLICABILITY TO DETECTING HEALTH CONDITIONS RELATED TO THREE COMMON DISEASE CATEGORIES: CARDIOVASCULAR DISEASES (CVD) [25], CHRONIC OBSTRUCTIVE PULMONARY DISEASE (COPD) [26], AND PARKINSON's/HunTINGTON's DisEASES (PD) [27], [28]. $\checkmark \checkmark$ INDICATES HIGH APPLICABILITY, $\checkmark$ INDICATES SOME APPLICABILITY, AND? INDICATES UNDETERMINED APPLICABILITY.

of the overall system illustrated in Fig. 1 and highlight the opportunities and challenges for each in integrating remote health monitoring into clinical practice.

\section{Data Acquisition And Sensing}

Physiological data is acquired by wearable devices that combine miniature sensors capable of measuring various physiological parameters, minor preprocessing hardware and a communications platform for transmitting the measured data. Table I summarizes various biomarkers that can be measured by current or soon-to-be-available wearable sensors. The level of applicability of these biomarkers to diagnosing four common disease categories is also indicated in the table.

The wearability requirement, poses physical limitations on the design of the sensors. The sensors must be light, small, and should not hinder a patient's movements and mobility. Also, because they need to operate on small batteries included in the wearable package, they need to be energy efficient. Though the battery may be rechargeable or replaceable, for convenience and to ensure that data is not lost during recharging or battery replacement periods, it is highly desirable that they provide extended durations of continuous operation without requiring charging or replacement.

The low energy operation requirement can also pose a challenge for the quality of the data captured in terms of the achievable signal to noise ratio. Recent designs [5], [29], [30] of flexible sensors that can be placed in contact with the skin in different body parts are particularly attractive for medical applications because, compared to alternatives, the close contact with the skin allows measurement of more physiological parameters and with greater accuracy. There have also been efforts to prolong the operational lifetime of wearable sensors by incorporating low power device and circuit level techniques [31], [32] and energy harvesting methods [33] . Moreover, utilizing intelligent sensing methods on system level can further increase the operational longevity.

Energy efficient sensing mechanisms have been studied in the related context of wireless sensor networks (WSNs) that are used to sense physical phenomenon in a distributed fash- ion. Although the sensor deployment in our health monitoring system is more concentrated compared to WSNs, existing methods for WSNs can be revisited to suit our needs. The proposed energy efficient sensing approaches revolve around assigning sensing tasks to the nodes based on their relative distance so as to sense the maximum amount of physical information while minimizing the energy consumption by removing possible redundant sensing tasks [34], [35] and by allocation of tasks based on the energy availability at each sensor [36]-[40]. Similar mechanisms can be applied to our system by defining and using a dynamic context that is based on energy availability and the health condition of the patient. For example, as indicated in Table I, individually sensed biomarkers have different levels of applicability for specific health conditions. When energy is severely limited and the vulnerable condition of the patient mandates focus on a specific biomarker, the other sensors be powered off in order to extend the lifetime. An IoT based sensing architecture facilitates the implementation of such schemes for improving energy efficiency adaptively by allowing dynamic utilization of sensors based on the context. In conventional data acquisition systems where sensors passively transmit the gathered information, such intelligence and flexibility may not be achievable. Also by offloading the decision making process for sensing task assignment to the cloud, more sophisticated algorithms can be applied without requiring manual intervention by the patient to manipulate the sensors or the software on the data concentrator.

Energy limitation of these devices necessitates the use of suitable low power communication protocols, as the communication can account a significant part of the power consumption in sensing devices. ZigBee over IEEE 802.15.4 is commonly used in low rate WPANs (LR-WPANs) to support communication between low power devices that operate in personal operating space (POS) of approximately 10m [41]. ZigBee provides reliable mesh networking with extended battery life. Bluetooth low energy (BLE) is another wireless communication protocol suitable for low power short range communication suitable for the unique requirements of applications such as health monitoring, sports, and home entertainment. The original Bluetooth protocol (IEEE 802.15.1) was designed to support relatively short range communication for applications of a streaming nature, such as audio. BLE modifies the framework by utilizing much longer sleep intervals to decrease the overall energy consumption. BLE achieves higher energy efficiency in terms of number of bytes sent per Joule of energy [42]. When using the aforementioned communication protocols, an intermediate node (data concentrator) is necessary to make sensors data and control accessible through Internet. To further realize the IoT concept, IPv6 over Low Power Wireless Personal Area Networks (6LoWPAN) has been proposed to seamlessly connect energy constrained WPAN devices to the Internet [19]. 6LoWPAN defines fragmentation techniques to fit IPv6 datagrams into IEEE 802.15.4 limited frame size to provide IP access to low power, low complexity sensing devices. 


\section{Cloud Data Storage and Processing}

Data aggregated by the concentrator needs to be transferred to the cloud for long term storage. Offloading data storage to the cloud offers benefits of scalability and accessibility on demand, both by patients and clinical institutions. Also, utilized with analytics and visualization (described in subsequent sections), cloud hosting and processing can reduce costs at HCOs and provide better diagnostic information. In this section, we outline such could architectures and discuss issues that impact long term medical data storage on the cloud.

a) Hybrid Cloud/Cloudlet Architecture: Cloudlet is a limited resource computing and storage platform that eliminates the need to outsource resource intensive tasks to the enterprise cloud [43]-[45]. Cloudlet computing has been introduced as a potential solution to deliver low latency to time critical tasks for health monitoring applications via body area networks [46]. Communication between concentrator and cloudlet is realized through WiFi interface. Direct connection between these two entities reduces data transfer latency for time critical tasks on aggregated data. LTE access provided in concentrator can in turn be used for direct data transfer from the concentrator to the cloud bypassing the cloudlet, while exposing the data to the latency imposed by mobile network.

b) Context-Aware Concentration via Smart Devices: As previously indicated, smart phones can at as concentrators in IoT infrastructure as today's smart phones can use both LTE and WiFi as the backhaul network. Data aggregation can be carried on either in cloudlet (thorough the WiFi connection between concentrator and the cloudlet) or the cloud (LTE). In studies, the former compared with the latter, has been shown to provide ten times the throughput and to require only a tenth of the access time, and half the power [47], [48]. Aggregated data, however, needs to be finally be stored in the cloud to allow distributed access and reliable storage. To effectively partition data aggregation tasks between cloud and cloudlet, context aware concentration may be utilized. Context can account for the current and expected status of the patient. For example, when the patient is in a critical condition requiring time critical monitoring of biosensor data, data concentration may be the preferred choice.

c) Privacy of the Data Concentrator: Although personally identifiable information can be removed before transmitting sensed data information, the system is still prone to aggregate disclosure attacks that can infer information via pattern recognition approaches [49]. Context aware data concentration, while offering some benefits, may also make sensed information vulnerable to aggregate disclosure attacks by allowing intruder to infer a patient's health information through network traffic analysis from concentrator to mobile back haul. Standard encryption techniques can be employed to ensure security in such settings [50].

d) Secure Data Storage in the Cloud: Privacy is of tremendous importance when storing individual's electronic medical records on the cloud. According to the terms defined by Health Insurance Portability and Accountability Act (HIPAA), the confidential part of medical records has to be protected from disclosure. When the medical records are outsourced to the cloud for storage, appropriate privacy preserving measures need to be taken to prevent unauthorized parties from accessing the information. Secure cloud storage frameworks have therefore been proposed for use with sensitive medical records [51]-[53]. Secure medical data processing on the cloud remains a challenge.

\section{ANALYTICS}

Compared with the lab and office based measurements that are the workhorses of current clinical medical practice, wearable sensors can readily incorporate multiple physiological measurements and enable gathering of data with much finer temporal sampling over much longer longitudinal time scales. These rich datasets represent a tremendous opportunity for data analytics: machine learning algorithms can potentially recognize correlations between sensor observations and clinical diagnoses, and by using these datasets over longer durations of time and by pooling across a large user base, improve medical diagnostics. As in other big data applications, the use of analytics here can improve accuracy, allow earlier detection, enable personalization, and reduce cost by reducing expensive lab procedures that are unnecessary.

Analytics on wearable sensor data can conceptually utilize a wide-range of pattern recognition and machine learning techniques [54], that have matured significantly and are now commonly available as toolboxes in several software packages [55], [56]. Several challenges must, however, be overcome before analytics can be deployed on any meaningful scale. Some of these challenges are analogous too those in other big data problems where as others are unique to out setting. There are also, however, challenges unique to analytics with wearable sensor data and to the medical and clinical seeing that we're focused upon. We highlight a few of these:

- Firstly, conventional medical instrumentation evolves at a fairly slow pace. New equipment and measuring devices typically require regulatory approval and training of medical personnel, which limits the rate at which new innovations can be introduced. The pace of development in electronics, on the other hand, is much faster and dictated by economic considerations to closely align with the so called Moore's law. This implies that the wearable sensors represent a much more dynamically evolving set of measurement devices than conventional medical instrumentation and as new sensor modules are added, sensors are updated, or obsoleted, there is likely to be a heterogeneous mix in deployment at any point in time. Machine learning methods need further development to deal with such heterogeneous and constantly evolving sensory inputs.

Analytics on data from wearable sensors needs to cope with streaming data, inevitably missing data values, and data of varying dimensionality and semantics as sensor designs change over time. Learning tasks face a significant challenges operating in these environment, even though some advances have been made in this area 
with the recent emergence of Big Data applications with massive volumes of high-dimensional observations that are often available in a streaming mode. Sequential algorithms targeting online support vector machines (SVMs) have been developed both in primal [57] as well as dual domains [58], [59]. These algorithms, however, are not designed to deal with time-varying feature dimensionality, incomplete data vectors due to absent features or acquisition failures, which if not treated properly, can seriously impair classification performance. To cope with missing input data, it is possible to impute the absent values using linear or nonlinear functions of the available features, and then proceed with clairvoyant learning schemes based on full data. Imputation schemes such as substitution with zero, entry-wise mean, and weighted averaging of the K-nearest-neighbors are popular interpolators, along with other more sophisticated but also more costly ones that can be found in [60]-[62].

- Secondly, while sensor data is plentiful, it is completely untagged and needs to be associated with corresponding "ground truth semantics", i.e., physician diagnoses, in order to be usable in training machine learning algorithms. Requesting this as additional inputs from already overloaded physicians is, however, infeasible. Thus alternative creative methods are required for generating the training data for our setting. An attractive possibility here is the ability to leverage clinical records, which are also becoming more readily accessible through the deployment of e-Health records systems. Figure 2 illustrates this framework for data analytics: the prior sensor data with associated data from the clinical records, mined across many individuals, can form the basis for machine learning where the physician diagnoses that are already part of the clinical record provide the necessary semantic labeling of ground truth once they are appropriately temporally-aligned with the sensor data. The learned classification and regression methodologies can then be used with current data to inform the physician's current prognoses/diagnoses. The process can then be iterated. The advantage of creating the linkage with the clinical records is that ongoing clinical practice can provide training data for the machine learning with little or no additional burden on the physicians.

- Thirdly, the data inputs for inference are highly heterogeneous. The sensory data represent very different modalities. The demographic and historical information in the clinical records, although extremely informative for inference is fundamentally of a very different nature from the sensor data. This range of heterogeneity challenges conventional machine learning approaches that deal primarily with homogeneous data. Graphical models [63] that allow combination of heterogeneous inputs in a common framework are therefore likely to be helpful for inference in these settings, though these are also likely to require significant customization to be effective

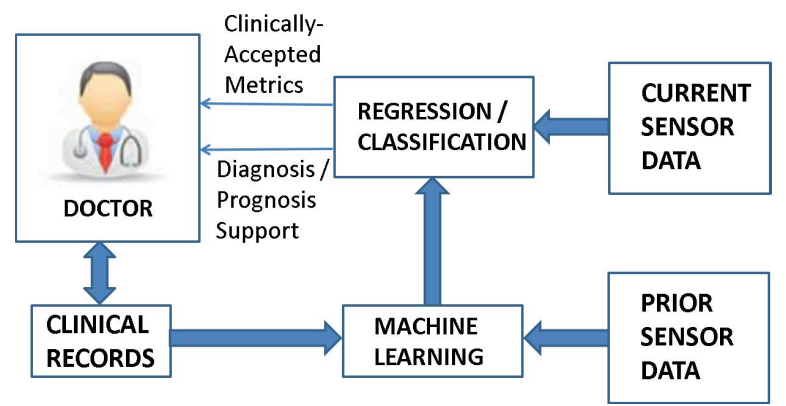

Fig. 2. Analytics workflow for systems integrating wearable sensor technology into clinical practice.

\section{VISUALIZATION}

It is impractical to ask physicians to pore over the voluminous data or analyses from IoT-based sensors. To be useful in clinical practice, the results from the Analytics Engine need to be presented to physicians in an intuitive format where they can readily comprehend the inter-relations between quantities and eventually start using the sensory data in their clinical practice. Visualization is recognized as an independent and important research area with a wide array of applications in both science and day to day life [64]. Given that color is a key discriminative attribute of our visual perception, it is unsurprising that color plays a key role in information visualization. Color distance and color category have been shown to be effective in allowing rapid identification and comprehension of differences in visually presented data [65]. The type of color visualization that is most effective is dependent on the type of data. For unidimensional relations, principles for visualization of relations have also been established: Qualitative palettes, where the same perceptual importance is given to all colors, are preferred when categorical data is transmitted, like bar graphs, pie charts, etc. Sequential palettes, where colors with lightness differences are assigned, are suggested for numerical variables whose value ranges in an interval. For classification maps, diverging palettes, that mix the qualitative and sequential palette instructions, are considered as the best option [66]. Visualization of multi-dimensional data, on the other hand, uses a combination of color, spatial location and other attributes and remains a challenging problem.

Data gathered or inferred from IoT sensors spans the complete spectrum of categories outlined in the previous paragraph and therefore an array of different visualization methodologies are required for effective use of the data. A distinct aspect of wearable sensor data, relative to data acquired at a laboratory or during a clinical visit, is that the data are gathered over a much longer longitudinal duration, with a finer temporal sampling, and simultaneously across multiple modalities. While the data represents a treasure trove for machine learning and inference, for the physician, it is problematic in the absence of tools to readily visualize and interact with the data. The time-varying and multi-dimensional aspects of the data pose a particular challenge because these have typically not been used in clinical practice even though the temporal variation 
and evolution of data and analyses results are of particular interest for diagnosis.

To highlight the utility of effective visualization of temporal information, we present a concrete example for cadio-vascular disease (CVD) monitoring [6], [67], [68]. Holter monitorbased ECG recording over durations of 24-48 hours is already utilized in CVD diagnosis [69]. Among other things, such monitoring is useful for detecting abnormal elongation of the QT interval, which represents the duration of time taken for electrical depolarization and repolarization of the ventricles and measured on the ECG as the duration between the start of the Q-wave and the end of the T-wave [70]. An abnormal prolongation of this interval, called Long QT Syndrome (LQTS) is an important indicator of potential malfunctioning of the heart [70], [71]. For diagnosis, a corrected value, QTc, that compensates for the natural variation in QT interval with the heart rate [72] is more directly informative than the raw QT values. The QTc interval is usually around $400 \mathrm{~ms}$ in a healthy person, and may go up to $500 \mathrm{~ms}$ or even higher with LQTS. After a patient undergoes a holter based recording session, QTc values are commonly obtained from analyses of ECG data and available to the physician for the duration of the study (one value per heart beat). A cardiologist that has 20 patients may have access to a table containing yesterday's two million QTc values, which can clearly not be individually examined as raw data. In current practice, cardiologists typically spotcheck about 10 seconds of the patient's ECG, and review the computed average values over a full 24-hour recording. This process discards a lot of key information. In the case of LQTS, for instance, QTc could be prolonged for several minutes or even hours without the doctor noticing the problem. There is a clinical need for a better way to visualize the full data set.

Figure 3 illustrates a scheme that we have recently developed for visualizing long-term monitoring of corrected QT (QTc) results [6], [67], [68]. The two plots shown in Figure 3 show 24 hour Holter monitoring results plotted within a circle. Midnight is the top of the plot and noon is the bottom. Low QTc values are inside the circle, and high values are along the edges. Different color bands are the QTc thresholds for normal (green: 360-425 ms), borderline (yellow: 450-500 $\mathrm{ms}$ ), and abnormal (red: $\geq 500 \mathrm{~ms}$ ). While the healthy patient's QTc (top figure) always stays within the normal region, the unhealthy patient's QTc (bottom figure) transitions into the abnormal region. These type of plots shown in Figure 3 allow for the physician to readily see and comprehend the the full temporal variation in QTc over the entire recording interval, instead of having to spot check individual values. Note also the significant change in QTc at night in Figure 3, which cannot be detected in clinical ECG monitoring done during the day. While this example focused on QTc and 24-hour observation periods, the process and framework will be similar to monitor other medical markers such as $\mathrm{O}_{2}$ saturation or glucose levels, and over different time intervals.

The preceding example illustrated the visualization of one parameter over the temporal duration of the recording via an informative image, which we note was static as opposed to
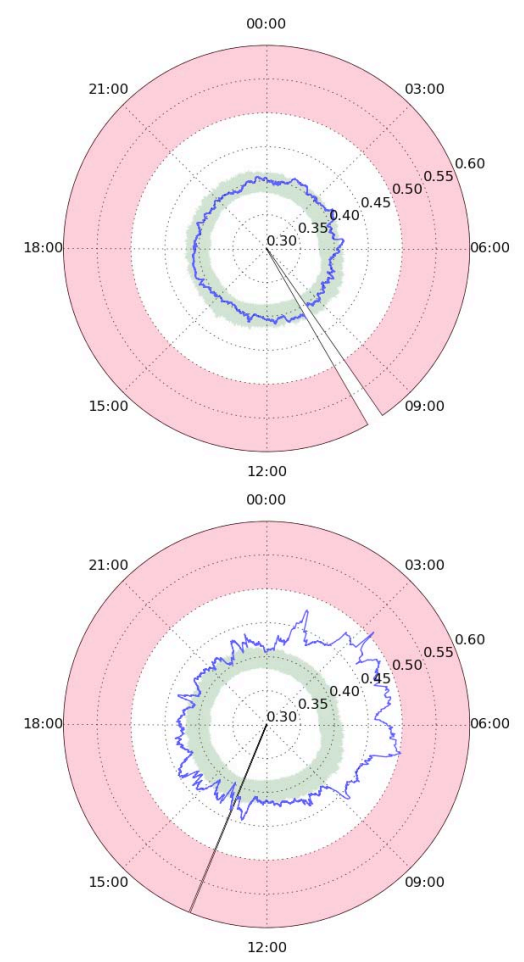

Fig. 3. QTc (in seconds) over 24 hours using the Bazett correction equation [73]. Top: healthy 24yo male patient. Bottom: 35yo male patient with the LQT2 genetic mutation, on beta blockers. "Slices" in the plots indicate a period that was not recorded. The green band is the interquartile range for healthy male patients in the THEW database [74]. Red represents abnormal and potentially dangerous QTc values.

varying with time. One can also readily present a number of parameters in parallel via one such image for each parameter. While this can be efficient for the physician to see the variation in each individual parameter at a glance, it is not as intuitive for understanding how the displayed parameters may co-vary over time. To address, this, additional visualization methods are required for visualizing the temporal dimension. The interactivity available through touch interfaces in modern mobile devices such as smartphones and tablets offers a particularly attractive opportunity for visualization of temporal relations. We have already been using such interactivity for improving the access of color deficient individuals to color imagery [75]. By leveraging interactivity, this interface also allows us to visualize changes over time, which as we already noted, is a particular aspect that makes wearable sensor data particularly useful but also challenging to use in physicians clinical settings. While there has been prior work on visualization of time-related data in biological settings [76], the focus has been entirely on static images suited for inclusion in a publication. Different from these, methodologies particularly adapted for interactive use that intuitively allows the physician to scroll back and forth in time to assess the temporal evolution and coevolution of different quantities of interest using smartphone and tablet devices already being deployed in their offices. 


\section{SUMMARY AND CONCLUDING REMARKS}

In this paper, we reviewed the current state and projected future directions for integration of remote health monitoring technologies into the clinical practice of medicine. Wearable sensors, particularly those equipped with IoT intelligence, offer attractive options for enabling observation and recording of data in home and work environments, over much longer durations than are currently done at office and laboratory visits. This treasure trove of data, when analyzed and presented to physicians in easy-to-assimilate visualizations has the potential for radically improving healthcare and reducing costs. We highlighted several of the challenges in sensing, analytics, and visualization that need to be addressed before systems can be designed for seamless integration into clinical practice.

\section{ACKNOWLEDGMENT}

This work was supported in part by the National Science Foundation grant CNS-1239423 and a gift from Nvidia Corp.

\section{REFERENCES}

[1] Jawbone Inc., "Jawbone fitness trackers," accessed April 2015. [Online]. Available: https://jawbone.com/up/trackers

[2] FitBit Inc., "flex: Wireless activity + sleep wristband," accessed April 2015. [Online]. Available: https://www.fitbit.com/flex

[3] Apple Inc., "Apple watch," accessed April 2015. [Online]. Available: https://www.apple.com/watch/

[4] A. Pantelopoulos and N. Bourbakis, "A survey on wearable sensor-based systems for health monitoring and prognosis," IEEE Trans. Sys., Man, and Cybernetics, Part C: Applic. and Reviews, vol. 40, no. 1, pp. 1-12, Jan 2010.

[5] D. Son, J. Lee, S. Qiao, R. Ghaffari, J. Kim, J. E. Lee, C. Song, S. J. Kim, D. J. Lee, S. W. Jun, S. Yang, M. Park, J. Shin, K. Do, M. Lee, K. Kang, C. S. Hwang, N. Lu, T. Hyeon, , and D.-H. Kim, "Multifunctional wearable devices for diagnosis and therapy of movement disorders," Nature Nanotechnology, pp. 1-8, 2014.

[6] A. Page, O. Kocabas, T. Soyata, M. Aktas, and J.-P. Couderc, "CloudBased Privacy-Preserving Remote ECG Monitoring and Surveillance," Annals of Noninvasive Electrocardiology (ANEC), 2014. [Online]. Available: http://dx.doi.org/10.1111/anec.12204

[7] R. Paradiso, G. Loriga, and N. Taccini, "A wearable health care system based on knitted integrated sensors," IEEE Trans. Info. Tech. in Biomedicine, vol. 9, no. 3, pp. 337-344, Sept 2005.

[8] A. Milenkovi, C. Otto, and E. Jovanov, "Wireless sensor networks for personal health monitoring: Issues and an implementation," Comput. Commun., vol. 29, no. 1314, pp. 2521 - 2533, 2006, wirelsess Senson Networks and Wired/Wireless Internet Communications. [Online]. Available: http://www.sciencedirect.com/science/article/pii/S0140366406000508

[9] M. Bazzani, D. Conzon, A. Scalera, M. Spirito, and C. Trainito, "Enabling the IoT paradigm in e-health solutions through the VIRTUS middleware," in IEEE 11th Int. Conf. on Trust, Security and Privacy in Computing and Com. (TrustCom), June 2012, pp. 1954-1959.

[10] A. Benharref and M. Serhani, "Novel cloud and SOA-based framework for E-Health monitoring using wireless biosensors," IEEE Journal of Biomed. and Health Inf., vol. 18, no. 1, pp. 46-55, Jan 2014.

[11] S. Babu, M. Chandini, P. Lavanya, K. Ganapathy, and V. Vaidehi, "Cloud-enabled remote health monitoring system," in Int. Conf. on Recent Trends in Inform. Tech. (ICRTIT), July 2013, pp. 702-707.

[12] C. Rolim, F. Koch, C. Westphall, J. Werner, A. Fracalossi, and G. Salvador, "A cloud computing solution for patient's data collection in health care institutions," in Second Int. Conf. on eHealth, Telemedicine, and Social Medicine, ETELEMED '10., Feb 2010, pp. 95-99.

[13] M. Lan, L. Samy, N. Alshurafa, M.-K. Suh, H. Ghasemzadeh, A. Macabasco-O'Connell, and M. Sarrafzadeh, "Wanda: An end-to-end remote health monitoring and analytics system for heart failure patients," in Proc. of the Conf. on Wireless Health, ser. WH'12. New York, NY, USA: ACM, 2012, pp. 9:1-9:8.
[14] L. Wei, N. Kumar, V. Lolla, E. Keogh, S. Lonardi, C. Ratanamahatana, and H. Van Herle, "A practical tool for visualizing and data mining medical time series," in Proc. 18th IEEE Symposium on Computer-Based Med. Sys., June 2005, pp. 341-346.

[15] Y. Mao, Y. Chen, G. Hackmann, M. Chen, C. Lu, M. Kollef, and T. Bailey, "Medical data mining for early deterioration warning in general hospital wards," in IEEE 11th Int. Conf. on Data Mining Workshops (ICDMW), Dec 2011, pp. 1042-1049.

[16] V. Ukis, S. Tirunellai Rajamani, B. Balachandran, and T. Friese, "Architecture of cloud-based advanced medical image visualization solution," in IEEE Int. Conf. on Cloud Computing in Emerging Markets (CCEM), Oct 2013, pp. 1-5.

[17] B. Rao, "The role of medical data analytics in reducing health fraud and improving clinical and financial outcomes," in Computer-Based Medical Systems (CBMS), 2013 IEEE 26th International Symposium on, June 2013, pp. 3-3.

[18] P. Ray, "Home health hub internet of things (H3IoT): An architectural framework for monitoring health of elderly people," in Int. Conf. on Science Eng. and Management Research (ICSEMR), Nov 2014, pp. 13.

[19] N. Bui and M. Zorzi, "Health care applications: A solution based on the internet of things," in Proc. of the 4th Int. Symposium on Applied Sciences in Biomed. and Com. Tech., ser. ISABEL '11. New York, NY, USA: ACM, 2011, pp. 131:1-131:5

[20] W. Zhao, C. Wang, and Y. Nakahira, "Medical application on internet of things," in IET Int. Conf. on Com. Tech. and Application (ICCTA 2011), Oct 2011, pp. 660-665.

[21] F. Hu, D. Xie, and S. Shen, "On the application of the internet of things in the field of medical and health care," in IEEE Int. Conf. on and IEEE Cyber, Physical and Social Computing Green Computing and Communications (GreenCom),(iThings/CPSCom), Aug 2013, pp. 2053 2058.

[22] T. Soyata, R. Muraleedharan, C. Funai, M. Kwon, and W. Heinzelman, "Cloud-Vision: Real-Time face recognition using a Mobile-CloudletCloud acceleration architecture," in Proceedings of the 17th IEEE Symposium on Computers and Communications (IEEE ISCC 2012), Cappadocia, Turkey, Jul 2012, pp. 59-66.

[23] G. Nalinipriya and R. Aswin Kumar, "Extensive medical data storage with prominent symmetric algorithms on cloud - a protected framework," in IEEE Int. Conf. on Smart Structures and Systems (ICSSS), March 2013, pp. 171-177.

[24] A. F. M. Hani, I. V. Paputungan, M. F. Hassan, V. S. Asirvadam, and M. Daharus, "Development of private cloud storage for medical image research data," in Int.Conf. on Computer and Inf. Sciences (ICCOINS), June 2014, pp. 1-6.

[25] "World health organization factsheeets: Cardiovascular diseases (CVDs)," online, accessed April 2015. [Online]. Available: http://www.who.int/mediacentre/factsheets/fs317/en/

[26] "World health organization factsheeets: Chronic obstructive pulmonary disease (COPD)," online, accessed April 2015. [Online]. Available: http://www.who.int/mediacentre/factsheets/fs315/en/

[27] "Parkinson's diseast information (mayo clinic website)," online, accessed April 2015. [Online]. Available: http://www.mayoclinic.org/ diseases-conditions/parkinsons-disease/basics/definition/con-20028488

[28] E. Dorsey, R. Constantinescu, J. Thompson, K. Biglan, R. Holloway, K. Kieburtz, F. Marshall, B. Ravina, G. Schifitto, A. Siderowf et al., "Projected number of people with Parkinson disease in the most populous nations, 2005 through 2030," Neurology, vol. 68, no. 5, pp. 384 386, 2007

[29] S. Xu, Y. Zhang, L. Jia, K. E. Mathewson, K.-I. Jang, J. Kim, H. Fu, X. Huang, P. Chava, R. Wang, S. Bhole, L. Wang, Y. J. Na, Y. Guan, M. Flavin, Z. Han, Y. Huang, and J. A. Rogers, "Soft microfluidic assemblies of sensors, circuits, and radios for the skin," Science, vol. 344, pp. 70-74, 2014

[30] D.-H. Kim, R. Ghaffari, N. Lu, and J. A. Rogers, "Flexible and stretchable electronics for biointegrated devices," Annual Review of Biomedical Engineering, pp. 113-128, 2012.

[31] O. Olorode and M. Nourani, "Reducing leakage power in wearable medical devices using memory nap controller," in Circuits and Sys. Conf. (DCAS), IEEE Dallas, Oct 2014, pp. 1-4.

[32] C. Park, P. Chou, Y. Bai, R. Matthews, and A. Hibbs, "An ultra-wearable, wireless, low power ecg monitoring system," in Biomed.l Circuits and Sys. Conf., BioCAS 2006. IEEE, Nov 2006, pp. 241-244. 
[33] T. Torfs, V. Leonov, C. Van Hoof, and B. Gyselinckx, "Body-heat powered autonomous pulse oximeter," in 5th IEEE Conf. on Sensors, Oct 2006, pp. 427-430.

[34] S. Madhani, M. Tauil, and T. Zhang, "Collaborative sensing using uncontrolled mobile devices," in Int. Conf. on Collaborative Computing: Networking, Applications and Worksharing, 2005, pp. 8 pp.-.

[35] C. T. Chou, R. Rana, and W. Hu, "Energy efficient information collection in wireless sensor networks using adaptive compressive sensing," in IEEE 34th Conf.on Local Computer Networks, LCN 2009., Oct 2009, pp. 443-450.

[36] H. Zhang and J. Hou, "Maintaining sensing coverage and connectivity in large sensor networks," Ad Hoc \& Sensor Wireless Networks, vol. 1, no. 1-2, pp. 89-123, 2005.

[37] C. Huang and Y. Tseng, "The Coverage Problem in a Wireless Sensor Network," Mobile Networks and Applications, vol. 10, no. 4, pp. 519528, 2005.

[38] Y. Chen and Q. Zhao, "On the lifetime of wireless sensor networks," IEEE Commun. Lett., vol. 9, no. 11, pp. 976-978, 2005.

[39] M. Cardei and J. Wu, "Energy-efficient coverage problems in wireless ad-hoc sensor networks," Computer Communications, vol. 29, no. 4, pp. 413-420, 2006.

[40] C. Yu and G. Sharma, "Camera scheduling and energy allocation for lifetime maximization in user-centric visual sensor networks," IEEE Trans. Image Proc., vol. 19, no. 8, pp. 2042-2055, Aug. 2010.

[41] J.-S. Lee, Y.-W. Su, and C.-C. Shen, "A comparative study of wireless protocols: Bluetooth, uwb, zigbee, and wi-fi," in 33rd Annual Conf. of the IEEE Indust. Elect. Society, IECON 2007., Nov 2007, pp. 46-51.

[42] M. Siekkinen, M. Hiienkari, J. Nurminen, and J. Nieminen, "How low energy is bluetooth low energy? comparative measurements with zigbee/802.15.4," in Wireless Communications and Networking Conference Workshops (WCNCW), 2012 IEEE, April 2012, pp. 232-237.

[43] Y. Li and W. Wang, "The unheralded power of cloudlet computing in the vicinity of mobile devices," in IEEE Globecom Workshops (GC Wkshps), Dec 2013, pp. 4994-4999.

[44] M. Kwon, Z. Dou, W. Heinzelman, T. Soyata, H. Ba, and J. Shi, "Use of network latency profiling and redundancy for cloud server selection," in Proceedings of the 7th IEEE International Conference on Cloud Computing (IEEE CLOUD 2014), Alaska, USA, Jun 2014, pp. 826832.

[45] T. Soyata, R. Muraleedharan, S. Ames, J. H. Langdon, C. Funai, M. Kwon, and W. B. Heinzelman, "Combat: mobile cloud-based compute/communications infrastructure for battlefield applications," in Proceedings of SPIE, vol. 8403, May 2012, pp. $84030 \mathrm{~K}-84030 \mathrm{~K}$

[46] M. Quwaider and Y. Jararweh, "Cloudlet-based for big data collection in body area networks," in 8th International Conference for Internet Technology and Secured Transactions (ICITST), Dec 2013, pp. 137-141.

[47] Y. Jararweh, L. Tawalbeh, F. Ababneh, and F. Dosari, "Resource efficient mobile computing using cloudlet infrastructure," in IEEE Ninth International Conference on Mobile Ad-hoc and Sensor Networks (MSN), Dec 2013, pp. 373-377.

[48] T. Soyata, H. Ba, W. Heinzelman, M. Kwon, and J. Shi, "Accelerating mobile cloud computing: A survey," in Communication Infrastructures for Cloud Computing, H. T. Mouftah and B. Kantarci, Eds. Hershey, PA, USA: IGI Global, Sep 2013, ch. 8, pp. 175-197.

[49] A. Abbas and S. Khan, "A review on the state-of-the-art privacypreserving approaches in the e-health clouds," IEEE Journal of Biomedical and Health Informatics, vol. 18, no. 4, pp. 1431-1441, July 2014

[50] National Institute of Standards and Technology, "Advanced encryption standard (AES)," Nov. 2001, fIPS-197.

[51] M. Li, S. Yu, Y. Zheng, K. Ren, and W. Lou, "Scalable and secure sharing of personal health records in cloud computing using attributebased encryption," IEEE Trans. Parallel Distrib. Syst., vol. 24, no. 1, pp. 131-143, Jan. 2013

[52] S. Ruj, M. Stojmenovic, and A. Nayak, "Privacy preserving access control with authentication for securing data in clouds," in Proceedings of the 2012 12th IEEE/ACM International Symposium on Cluster, Cloud and Grid Computing (Ccgrid 2012), ser. CCGRID '12. Washington, DC, USA: IEEE Computer Society, 2012, pp. 556-563.

[53] M. Li, S. Yu, Y. Zheng, K. Ren, and W. Lou, "Scalable and secure sharing of personal health records in cloud computing using attribute-based encryption," IEEE Transactions on Parallel and Distributed Systems, vol. 24, no. 1, pp. 131-143, 2013.

[54] C. Bishop, Pattern recognition and machine learning. New York, NY: Springer, 2006
[55] Mathworks Inc., MATLAB The Language of Technical Computing, Natick, Massachusetts, accessed April 2015. [Online]. Available: http://www.mathworks.com/products/matlab/

[56] R Core Team, R: A Language and Environment for Statistical Computing, R Foundation for Statistical Computing, Vienna, Austria, accessed April 2015. [Online]. Available: http://www.R-project.org/

[57] S. Shalev-Shwartz, Y. Singer, N. Srebro, and A. Cotter, "Pegasos: Primal estimated sub-gradient solver for SVM," Mathematical Programming, vol. 127, no. 1, pp. 3-30, 2011.

[58] A. Bordes, S. Ertekin, J. Weston, and L. Bottou, "Fast kernel classifiers with online and active learning," J. of Machine Learning Research, vol. 6, pp. 1579-1619, 2005

[59] J. C. Platt, "Fast training of support vector machines using sequential minimal optimization," Advances in Kernel Methods, pp. pp. 185-208, 1999.

[60] G. Chechik, G. Heitz, G. Elidan, P. Abbeel, and D. Koller, "Max-margin classification of data with absent features," J. of Machine Learning Research, vol. 9, pp. 1-21, 2008

[61] P. J. Garcia-Laencina, J. L. Sancho-Gomez, and A. R. Figueiras-Vidal, "Pattern classification with missing data: A review," J. of Machine Learning Research, vol. 19, no. 2, pp. 263-282, 2010.

[62] R. J. A. Little and D. B. Rubin, Statistical Analysis with Missing Data. New Jersey: Wiley, 2002.

[63] M. Jordan and T. Sejnowski, Eds., Graphical models: foundations of neural computation, ser. Computational neuroscience. MIT Press, 2001.

[64] E. R. Tufte and P. Graves-Morris, The visual display of quantitative information. Graphics press Cheshire, CT, 1983, vol. 2.

[65] C. Healey, "Choosing effective colours for data visualization," in Proceedings of the 7th conference on Visualization. IEEE, 1996, pp. 263 270.

[66] L. D. Bergman, B. E. Rogowitz, and L. A. Treinish, "A rule-based tool for assisting colormap selection," in Proceedings of the 6th conference on Visualization. Washington, DC, USA: IEEE Computer Society, 1995, pp. 118-.

[67] Mehmnet Aktas and Alex Page and Tolga Soyata and JP Couderc "Incidence of diurnal QT concealment in genotype positive long QT 1 and 2 patients," in Heart Rhythm Society's Annual Scientific Sessions, 2015, to be presented, May 13-16, 2015, Boston, MA

[68] O. Kocabas, T. Soyata, J.-P. Couderc, M. Aktas, J. Xia, and M. Huang, "Assessment of cloud-based health monitoring using homomorphic encryption," in Proceedings of the 31st IEEE International Conference on Computer Design (ICCD), Ashville, VA, USA, Oct 2013, pp. 443 446.

[69] "Holter monitor," online, accessed April 2015. [Online]. Available: http://www.nlm.nih.gov/medlineplus/ency/article/003877.htm

[70] "Long QT syndrome (mayo clinic website)," online, accessed April 2015. [Online]. Available: http://www.mayoclinic.org/ diseases-conditions/long-qt-syndrome/basics/causes/con-20025388

[71] H. Morita, J. Wu, and D. P. Zipes, "The QT syndromes: long and short," The Lancet, vol. 372, no. 9640, pp. 750 - 763, 2008. [Online]. Available: http://www.sciencedirect.com/science/article/pii/S0140673608613070

[72] L. S. Fridericia, "Die Systolendauer im Elektrokardiogramm bei normalen Menschen und bei Herzkranken," Acta Medica Scandinavica, vol. 53, pp. 469-486, 1920.

[73] H. C. Bazett, "An Analysis of Time Relations of the Electrocardiogram,' Heart, vol. 7, pp. 353-370, 1920.

[74] J. Couderc, "The telemetric and holter ECG warehouse initiative (THEW): A data repository for the design, implementation and validation of ecg-related technologies," in Engineering in Medicine and Biology Society (EMBC), 2010 Annual International Conference of the IEEE. IEEE, 2010, pp. 6252-6255. [Online]. Available: http://www.thew-project.org/

[75] C. Lau, N. Perdu, C. E. Rodríguez-Pardo, S. Süsstrunk, and G. Sharma, "An interactive app for color deficient viewers," in Proc. SPIE: Color Imaging XX: Displaying, Processing, Hardcopy, and Applications, R. Eschbach, G. G. Marcu, and A. Rizzi, Eds., vol. 9395, Feb. 2015, pp. 939512,1-9.

[76] M. Secrier and R. Schneider, "Visualizing time-related data in biology, a review," Briefings in Bioinformatics, 2013. [Online]. Available: http //bib.oxfordjournals.org/content/early/2013/04/12/bib.bbt021.abstract 